\title{
Founder mutations in the Netherlands: familial idiopathic ventricular fibrillation and DPP6
}

\author{
P. G. Postema - I. Christiaans • N. Hofman - M. Alders • \\ T. T. Koopmann • C. R. Bezzina $\cdot$ P. Loh • \\ K. Zeppenfeld • P. G. A. Volders • A. A. M. Wilde
}

Published online: 22 April 2011

(C) The Author(s) 2011. This article is published with open access at Springerlink.com

\begin{abstract}
In this part of a series on founder mutations in the Netherlands, we review familial idiopathic ventricular fibrillation linked to the DPP6 gene. Familial idiopathic ventricular fibrillation determines an intriguing subset of the inheritable arrhythmia syndromes as there is no recognisable phenotype during cardiological investigation other than ventricular arrhythmias highly associated with sudden cardiac death. Until recently, it was impossible to identify presymptomatic family members at risk for fatal events. We uncovered several genealogically linked families affected by numerous sudden cardiac deaths over the past centuries, attributed to familial idiopathic ventricular fibrillation. Notably, ventricular fibrillation in these families was provoked by very short coupled monomorphic extrasystoles. We were able to associate their phenotype of lethal arrhythmic events with a haplotype harbouring the DPP6 gene. While this gene has not earlier been related to cardiac arrhythmias, we are now able, for the first time, to identify and to offer timely treatment to
\end{abstract}

P.G. Postema and I. Christiaans contributed equally to this work.

P. G. Postema • T. T. Koopmann • C. R. Bezzina • A. A. M. Wilde Department of Cardiology and Heart Failure Research Center, Academic Medical Center, University of Amsterdam,

Amsterdam, the Netherlands

I. Christiaans $\cdot$ N. Hofman $\cdot$ M. Alders

Department of Clinical Genetics, Academic Medical Center,

University of Amsterdam,

Amsterdam, the Netherlands

T. T. Koopmann

Department of Medical Physiology,

University Medical Center Utrecht,

Utrecht, the Netherlands

P. Loh

Department of Cardiology, University Medical Center Utrecht,

Utrecht, the Netherlands presymptomatic family members at risk for future fatal events solely by genetic analysis. Therefore, when there is a familial history of unexplained sudden cardiac deaths, a link to the DPP6 gene may be explored as it may enable risk evaluation of the remaining family members. In addition, when closely coupled extrasystoles initiate ventricular fibrillation in the absence of other identifiable causes, a link to the DPP6 gene should be suspected.

Keywords Sudden cardiac death .

Idiopathic ventricular fibrillation · DPP6

\section{Introduction}

Familial idiopathic ventricular fibrillation (IVF) can be regarded as the most difficult subset of the inheritable arrhythmia syndromes [1]. Unlike the other primary arrhyth-

\author{
K. Zeppenfeld \\ Department of Cardiology, Leiden University Medical Center, \\ Leiden, the Netherlands
}

P. G. A. Volders

Department of Cardiology, Cardiovascular Research Institute Maastricht, Maastricht University Medical Center, Maastricht, the Netherlands

\author{
A. A. M. Wilde $(\bowtie)$ \\ Department of Cardiology, Academic Medical Center, \\ Meibergdreef 9, \\ 1105 AZ Amsterdam, the Netherlands \\ e-mail: a.a.wilde@amc.uva.nl
}


mia syndromes (e.g. long QT syndrome, catecholaminergic polymorphic ventricular tachycardias and Brugada syndrome), there are no clinical signs of future risk for ventricular fibrillation (VF) other than a (family) history of (aborted) sudden cardiac arrest. The expression IVF best acknowledges our current inability to identify a plausible cause or substrate for the occurrence of VF in these patients who were previously considered healthy [2]. It is estimated that IVF accounts for 9000 to 12,500 cases per year in the USA on a total annual burden of sudden cardiac deaths (SCD) of approximately 180,000 to 250,000 [3-6]. Because there are also familial forms of IVF, the risk can be transferred from parents to children, and subsequently, whole families can be at risk for VF and SCD. Unfortunately, risk stratification to determine which of the remaining family members may develop potentially fatal cardiac arrhythmias has been impossible due to the lack of clinical abnormalities.

Recently, we have made a breakthrough in several Dutch families heavily affected by SCD attributed to familial IVF over the past centuries. We were able to associate their arrhythmic events with a single haplotype harbouring the DPP6 gene as a founder effect (i.e. the families are descendants from the same ancestor) [7]. Because this gene has not earlier been related to cardiac arrhythmias, we are now able, for the first time, to identify presymptomatic family members at risk for future fatal events solely by genetic analysis and treat them accordingly. Here, we review what we have learned from these families and discuss the possible mechanisms underlying this form of familial IVF linked to the DPP6 gene.

\section{First clinical contacts with the family}

Three separate presentations to our cardiogenetics outpatient clinic finally resulted in the recognition of their familial connection and ultimately initiated a successful gene identification study to the culprit genetic abnormality.

\section{Case 1}

A 44-year-old woman with an unremarkable medical history visited our outpatient clinic because of the SCD of her younger brother and her younger sister. One day, at the age of 31 years, her previously healthy brother was unexpectedly found dead in bed, and post-mortem evaluation did not reveal a cause of death. Several years later, her sister was found dead in bed at the same age as her deceased brother, and also, she had previously been considered healthy. Notably, this sister consulted a cardiologist because of palpitations 1 month before her death. On cardiac examination, she had one non-sustained ventricular tachycardia on 24-h Holter monitoring, but her ECG, echocardiography and exercise test were normal. Her ECG is shown in Fig. 1a. The family history further revealed that an uncle died unexpectedly at the age of 48 years late in the evening while he was out to inspect his cattle, and a great grandfather also died suddenly at a young age. Because of this impressive family history, the 44-year-old woman underwent a cardiac examination, which was unremarkable. Her ECG is shown in Fig. 1b. Because no abnormalities were found, it
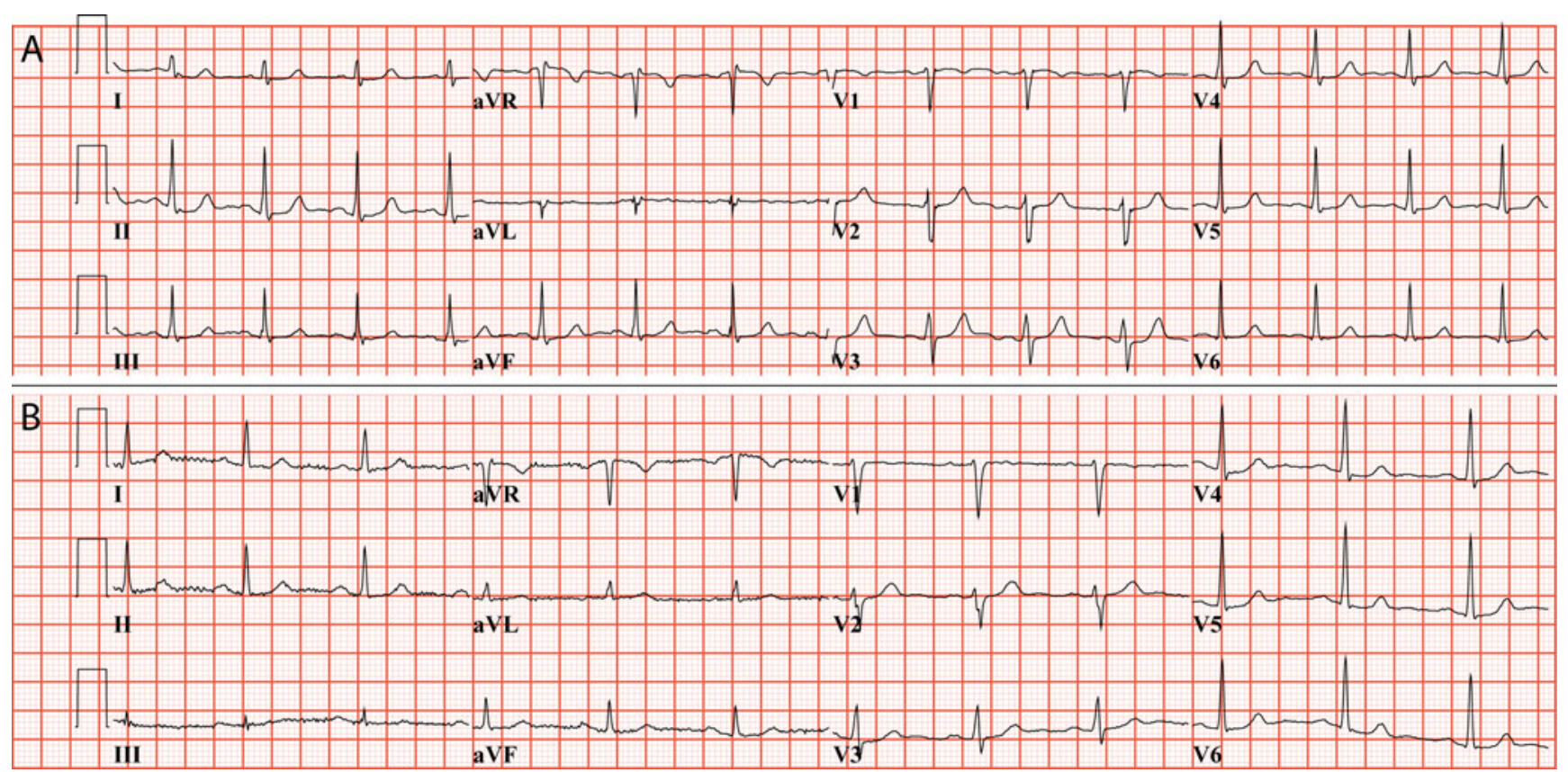

Fig. 1 ECGs of two sisters, both of whom experienced sudden cardiac arrest. a ECG recorded only 1 month before sudden cardiac death at the age of 31 years while in bed. $\mathbf{b}$ ECG recorded only a few months before resuscitation from IVF at the age of 44 years, while drinking coffee. ECG a, courtesy of Dr. M.P. van den Berg, University Medical Center Groningen, the Netherlands 
was discussed that prophylactic treatment was not readily indicated, but her family members were asked to visit a cardiologist in a search for an inheritable arrhythmia syndrome. A few months later, while further cardiac examinations of her two remaining brothers were carried out, she suffered from VF while drinking coffee in the morning. Fortunately, she was successfully resuscitated. Subsequently, an implantable cardioverter-defibrillator (ICD) was implanted, which to date has not discharged. However, only a few months after her resuscitation, her nephew (her deceased brother's son) was also resuscitated from VF at the age of 16 years while climbing stairs. After an unremarkable complete cardiac examination, he too had an ICD implanted, and he has received appropriate discharges on several VF recurrences in the past years.

\section{Case 2}

The 69-year-old father of a family contacted our outpatient clinic because of the SCD of two of his sons. The youngest son died in his sleep at the age of 32, and the oldest son died at the age of 37 years while sitting in a car (in the passenger seat). Both were previously considered healthy. Post-mortem evaluations did not reveal the cause of these young deaths. Further family history revealed that the father had three uncles who had also died unexpectedly in bed. They were 41, 38 and 28 years old, respectively. As the father is the link between his sons and his uncles, he was considered to have transmitted the SCD-related gene defect although he had been asymptomatic his whole life. Complete cardiac evaluation of the father and his eight remaining children (ECG, echocardiography, 24-h Holter and exercise test) did not reveal abnormalities other than pulmonary emphysema in the father. Three of his children chose to have an ICD implanted for primary prevention of SCD. They all remained free of arrhythmias until now. However, at the age of 74 , the father died from a noncardiac cause.

\section{Case 3}

A 33-year-old male was admitted to our hospital after his successful resuscitation. He collapsed after he had just gone to bed. Besides several unexplained syncopes in the months preceding the resuscitation, he had an unremarkable medical history, and complete cardiac examination (ECG, echocardiography, 24-h Holter and exercise test) revealed no abnormalities. An ICD was implanted, which appropriately discharged on several VF recurrences during the last years. Further history taking revealed that two previously considered healthy nephews of his great grandmother had died suddenly and unexpectedly, both at the age of 30 years, one while walking and the other while cycling.

\section{Gene finding study}

Classical linkage analyses in familial IVF has previously been impossible due to the lack of a phenotype which indicates affected carriers, while those who are affected are only recognised because of a sudden cardiac arrest which most often results in death. This scenario often leaves too little clinical and genetic information to perform successful studies in a search for the culprit gene. However, genealogical analyses revealed that the three families mentioned above were in fact linked many generations before. As we now recognised that they probably belonged to one family, we were also able to start an identity-by-descent genome-wide haplotype-sharing analysis. The whole genome of eight family members was investigated by labelling over 300,000 common genetic variants (also known as single nucleotide polymorphisms or SNPs) with the use of Illumina HumanHap BeadChips. This enabled construction of shared segments (haplotypes) between the affected family members. As a result, one large haplotype of 301 contiguous SNPs was found on chromosome 7, which was highly significantly shared between affected family members of the three families (Fig. 2). This strongly suggested that the culprit gene for IVF in this family was located within this shared region. At this stage, the segment contained four genes: HTR5A (serotonin receptor in brain), PAX-IP1 (involved in DNA damage response), $A C T R 3 B$ (expressed in tumorigenic cell lines) and DPP6 (associated with potassium channel subunits). Particularly, the last mentioned, DPP6, was interesting as it encodes for a putative subunit of the ion channel involved in the transient outward current $\left(I_{\mathrm{to}}\right)$ in heart [8]. However, direct sequencing of all the DPP6 coding exons in the affected family members did not reveal a mutation. Instead, a base pair substitution $(\mathrm{C}>\mathrm{T})$ was found just outside the coding region, upstream of one of the initiation codons. This variant was not documented in 350 controls but could not be directly marked as pathogenic.

To determine the relevance of DPP6 in familial IVF, we then screened 42 IVF probands considered unrelated to the three families of our initial studies for the same variant in DPP6. In seven families, we indeed identified the same $\mathrm{C}>\mathrm{T}$ variant in DPP6. This indicates that they are in fact descendants from the same ancestors, which was confirmed by further extensive genealogical research for most families. Further analyses of the risk haplotype on chromosome 7 in these additional families allowed us to redefine the shared region between the affected family members. Subsequently, the haplotype became smaller and now excluded HTR5A, $P A X-I P 1$ and $A C T R 3 B$. Thus, DPP6 was the only gene in the remaining shared region. Meanwhile, we identified another 
Fig. 2 Shared genetic regions between affected family members. SNPS single nucleotide polymorphisms. The arrow indicates the largest shared genetic region on chromosome $7 \mathrm{q} 36$

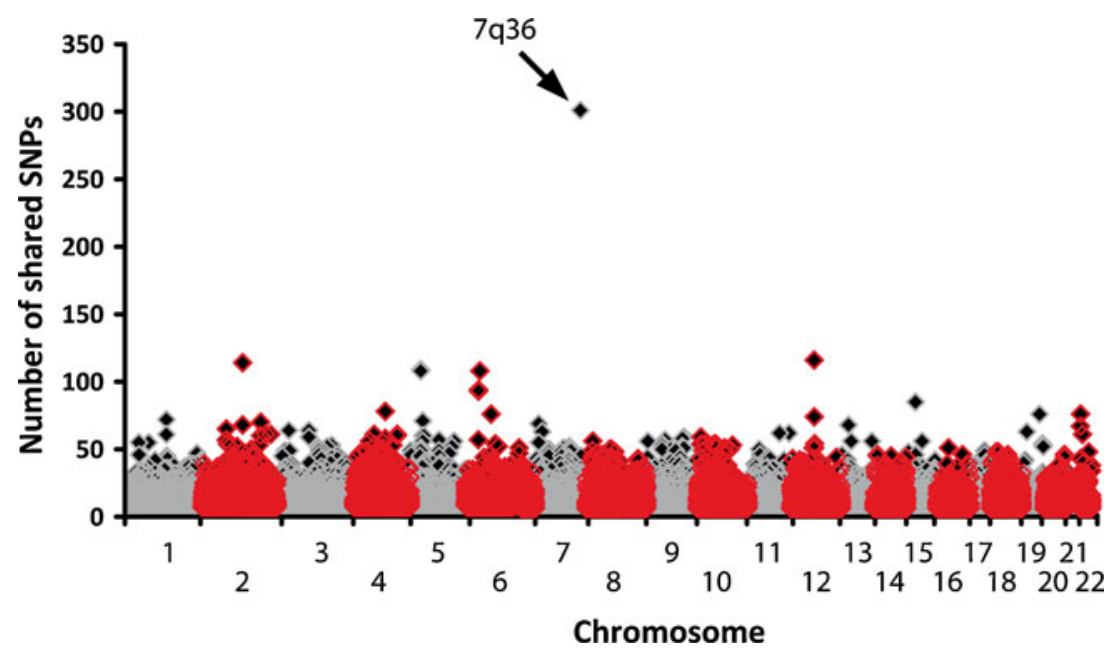

three probands harbouring this genetic defect, all of them from families with a high prevalence of SCD.

Although we did not document an exon mutation in $D P P 6$, we know that regulation of gene expression might occur outside the coding region. Therefore, we set out to identify differences in DPP6 expression between affected family members and controls which could further establish a role for DPP6 in IVF. Heart biopsies were taken from five risk haplotype carriers and compared with biopsies from controls. Indeed, average mRNA expression levels of DPP6 were increased 22-fold in haplotype carriers as compared with controls, which indicates the overexpression of DPP6. Additionally, we excluded changed expression of the neighbouring genes $P A X-1 P$ and $A C T R 3 B$.

\section{Further clinical evaluation of DPP6 haplotype carriers}

As we had now identified a risk haplotype on chromosome 7 (which contains the DPP6 gene) to be associated with
IVF in these families, we could determine which family members are at risk for IVF.

Currently, we already have clinical data of 255 family members of whom 117 carry the risk haplotype. As this is a founder 'mutation', the family members are unevenly distributed over the country with highest prevalence in the centre, around the cities of Woerden and Gouda (Fig. 3). Of course, because of migration, we also identified haplotype carriers in other parts of the country. This DPP6 founder mutation now almost reaches the size of another very large founder mutation, which is associated with cardiac arrests based on a mutation in the cardiac sodium channel gene SCN5A $[9,10]$.

Survival in affected family members is significantly decreased as can be appreciated from Fig. 4. At the age of 60 years, already $50 \%$ of the risk haplotype carriers have died or experienced a sudden cardiac arrest. As to the ventricular arrhythmias that affect this family, recordings show that these are elicited by very short coupled (right) ventricular extrasystoles (e.g. Fig. 5).
Fig. 3 Distribution of the $D P P 6$ family in the Netherlands. The founder effect can be appreciated by the highest prevalence in the centre of the country. a Distribution of index patients. $\mathbf{b}$ Distribution of all the family members. Colours indicate number of individuals

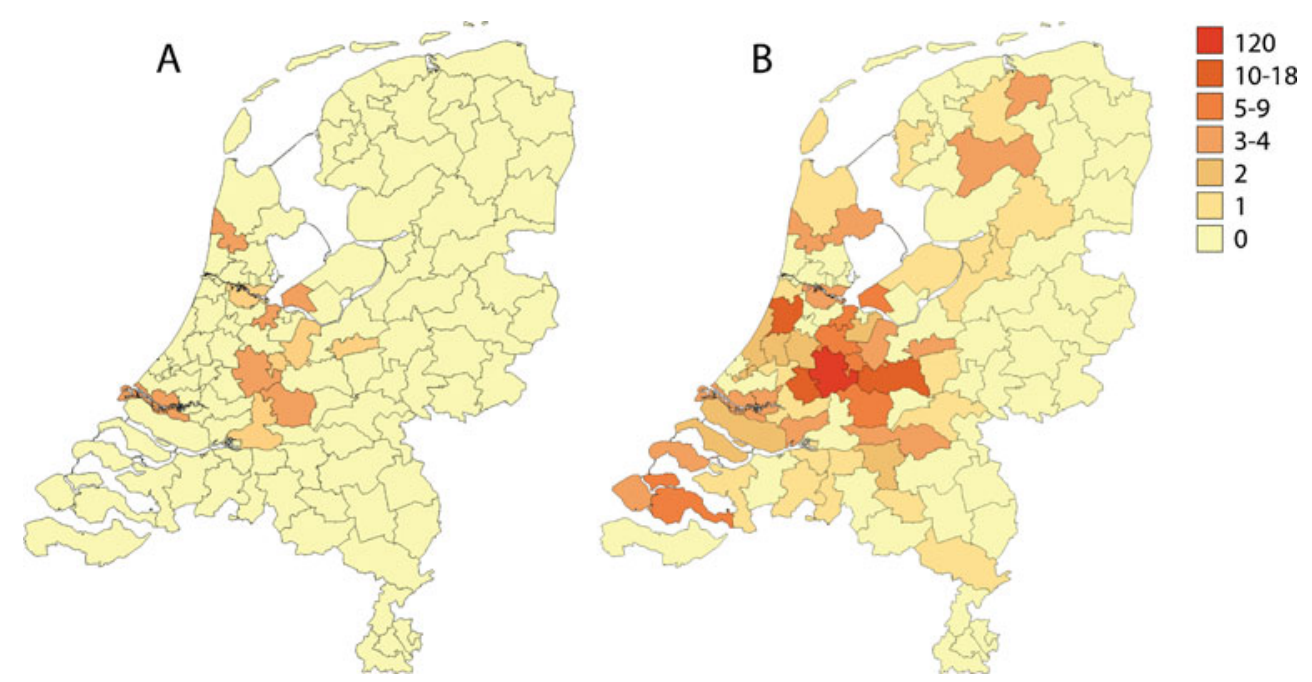


Fig. 4 Survival plot showing mortality or cardiac arrest. Median survival (50\%) for risk haplotype carriers is located at 60 years of age

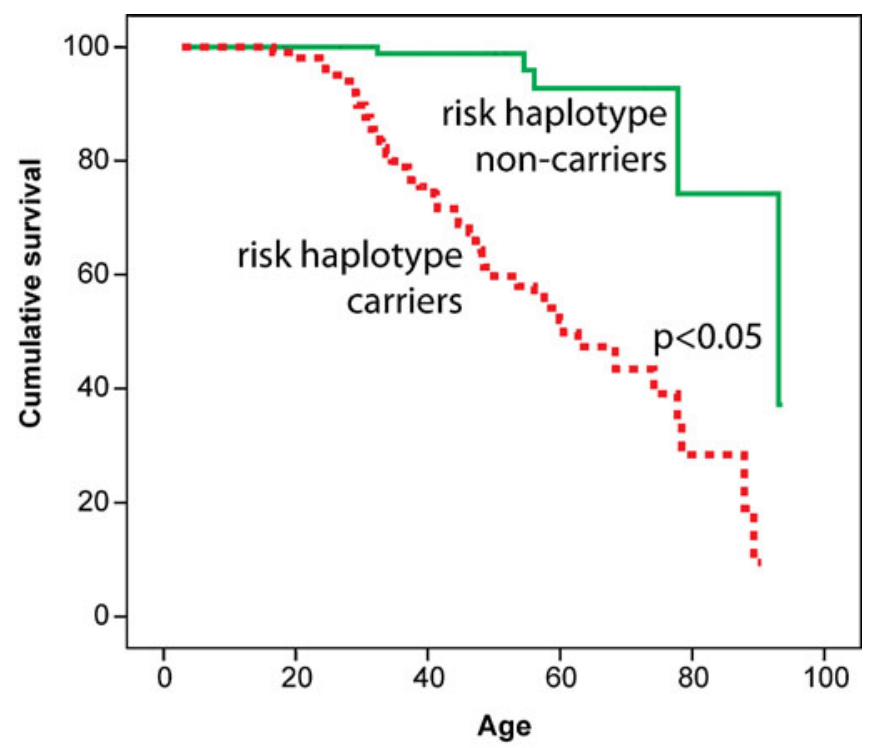

Number at risk

Risk haplotype non-carriers $138 \quad 110$ Risk haplotype carriers

117
69

61
24
24

4

0

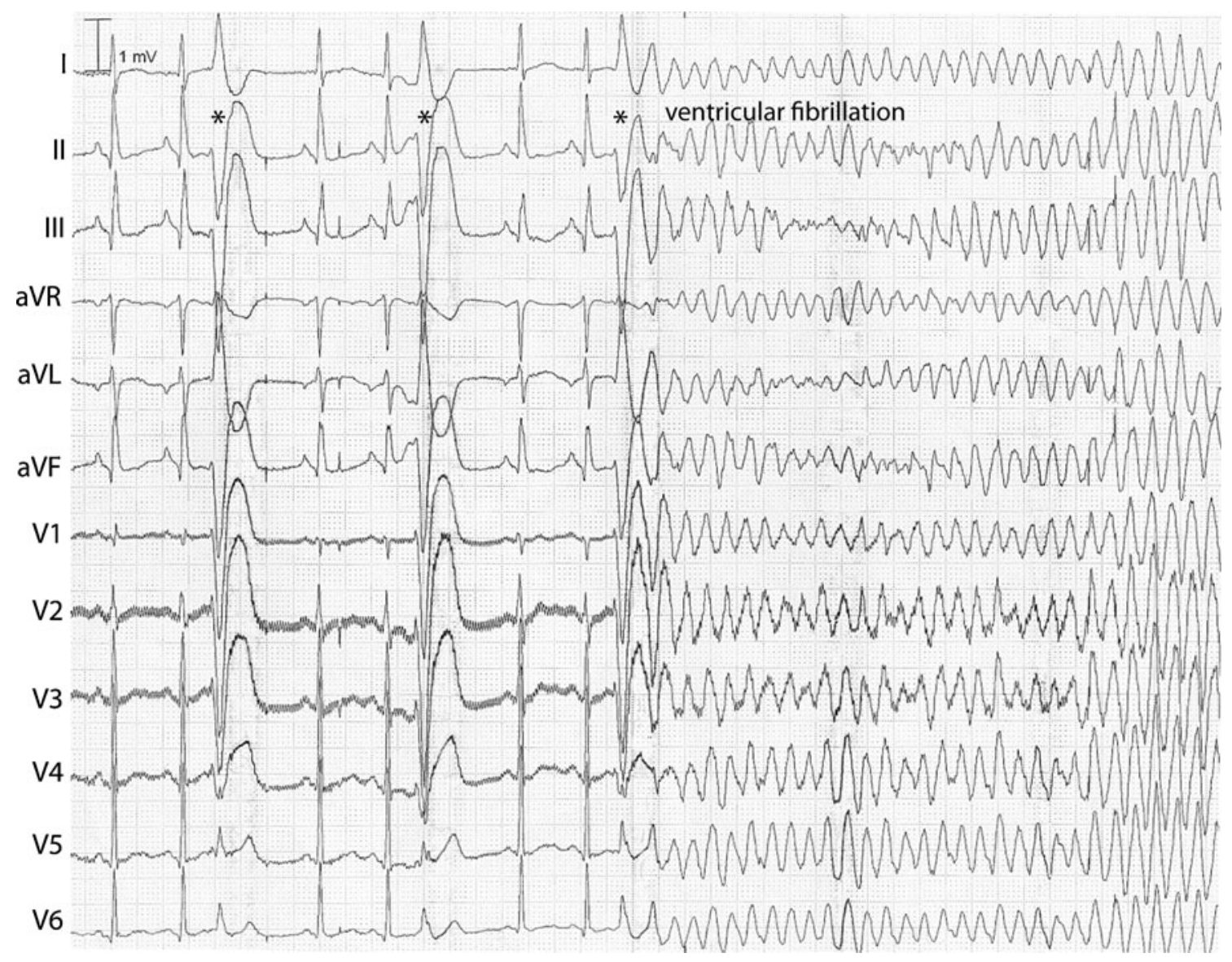

Fig. 5 ECG recording of idiopathic ventricular fibrillation (IVF) in a risk haplotype carrier. The short coupled ventricular extrasystoles from the right ventricular apex/lower free wall (indicated by asterisks) first result in compensatory pauses and then in IVF requiring external defibrillation 


\section{Treatment}

At this moment, we can only determine the risk of IVF in asymptomatic family members by carrier status of the risk haplotype on the DPP6 gene. Because this is such a malignant phenotype, and while we do not have pharmacological treatment to eradicate the risk of IVF, we now offer ICDs to risk haplotype carriers between approximately 20 and 50 years. These cutoffs were determined on pedigree analyses which did not show an increased risk for sudden unexplained cardiac arrest/death before and after these age boundaries as compared with the general Dutch population (standardised mortality ratio) [11]. Although this strategy should prevent sudden cardiac deaths due to termination of IVF by the ICD, we are well aware that we also introduce a large burden of (serious) ICD-related complications to these young persons [12]. In one of the severely symptomatic patients, quinidine has been effective in preventing VF recurrence. This closely follows previous favourable experiences of quinidine in IVF [13]. In another severely affected patient, the quinidine effect was only temporary, however. Also, the experience with ablation therapy in IVF is currently limited [14], but has led to temporary success in one of the patients.

\section{Mechanisms and further studies}

The mechanism by which DPP6 overexpression would result in IVF without discernable ECG abnormalities is currently unknown. As mentioned, DPP6 is putatively involved in the transient outward potassium current $I_{\text {to }}$ in heart [8]. This current is active in phase 1 of the cardiac action potential. While DPP6 mRNA seems to be similarly expressed between epicardium and endocardium, DPP6 overexpression does not necessarily have to alter the transmural $I_{\text {to }}$ gradient. The latter may explain the absence of discernable ECG abnormalities.

Our future focus on this family and its members' vulnerability to IVF has three directions. First, further clinical studies are deployed to further characterise the affected versus the non-affected family members by detailed ECG and imaging studies. When available, we also plan to perform detailed histological studies on cardiac tissue. Second, further characterisation of the exact derangement in the risk haplotype is pursued. And third, we are working on in vitro studies to recapitulate the effect of DPP 6 derangements on the cardiac action potential to be able to better understand its arrhythmogenic potential.

\section{Summary}

A new arrhythmia gene on chromosome $7 \mathrm{q} 36, D P P 6$, is linked to familial IVF and shows a very malignant phenotype. This phenotype consists of cardiac arrest by IVF at young age, which is elicited by short coupled extrasystoles. While this gene has not earlier been related to cardiac arrhythmias, we are now able, for the first time, to identify presymptomatic family members at risk for future fatal events solely by genetic analysis. Affected patients can be offered preventive treatment accordingly. Therefore, when there is a family history of unexplained sudden cardiac deaths, we make the reader aware of this particular link to the DPP6 gene as it may enable risk stratification of the remaining family members. In addition, when closely coupled extrasystoles initiate ventricular fibrillation in the absence of other identifiable causes, a link to the DPP6 gene should also be suspected.

Acknowledgements We would like to thank the family members for their cooperation, Sophie van Nes for her efforts in collecting patient data, all who have worked on this project and all referring physicians are gratefully acknowledged.

Funding This research is financially supported by ZorgOnderzoek Nederland (ZonMw), grant number 62000010 and the Netherlands Heart Foundation (2009B066).

Open Access This article is distributed under the terms of the Creative Commons Attribution Noncommercial License which permits any noncommercial use, distribution, and reproduction in any medium, provided the original author(s) and source are credited.

\section{References}

1. Viskin S, Belhassen B. Idiopathic ventricular fibrillation. Am Heart J. 1990;120:661-71.

2. Survivors of out-of-hospital cardiac arrest with apparently normal heart. Need for definition and standardized clinical evaluation. Consensus Statement of the Joint Steering Committees of the Unexplained Cardiac Arrest Registry of Europe and of the Idiopathic Ventricular Fibrillation Registry of the United States. Circulation. 1997;95:265-272.

3. Chugh SS, Reinier K, Teodorescu C, et al. Epidemiology of sudden cardiac death: clinical and research implications. Prog Cardiovasc Dis. 2008;51:213-28

4. Zipes DP, Wellens HJ. Sudden cardiac death. Circulation. 1998;98:2334-51.

5. Huikuri HV, Castellanos A, Myerburg RJ. Sudden death due to cardiac arrhythmias. N Engl J Med. 2001;345:1473-82.

6. Chugh SS, Jui J, Gunson K, et al. Current burden of sudden cardiac death: multiple source surveillance versus retrospective death certificate-based review in a large U.S. community. J Am Coll Cardiol. 2004;44:1268-75.

7. Alders M, Koopmann TT, Christiaans I, et al. Haplotype-sharing analysis implicates chromosome 7q36 harboring DPP6 in familial idiopathic ventricular fibrillation. Am J Hum Genet. 2009;84:468-76.

8. Radicke S, Cotella D, Graf EM, et al. Expression and function of dipeptidyl-aminopeptidase-like protein 6 as a putative betasubunit of human cardiac transient outward current encoded by Kv4.3. J Physiol. 2005;565:751-6.

9. Postema PG, van den Berg MP, van Tintelen JP, et al. Founder mutations in the Netherlands. SCN5a 1795insD, the first described 
arrhythmia overlap syndrome and one of the largest and best described characterised families worldwide. Neth Heart J. 2009;17:422-8.

10. van den Berg MP, Wilde AA, Viersma JW, et al. Possible bradycardic mode of death and successful pacemaker treatment in a large family with features of long QT syndrome type 3 and Brugada syndrome. J Cardiovasc Electrophysiol. 2001;12:630-6.

11. Sijbrands EJ, Westendorp RG, Defesche JC, et al. Mortality over two centuries in large pedigree with familial hypercholesterolaemia: family tree mortality study. BMJ. 2001;322:1019-23.
12. Tung R, Zimetbaum P, Josephson ME. A critical appraisal of implantable cardioverter-defibrillator therapy for the prevention of sudden cardiac death. J Am Coll Cardiol. 2008;52:1111-21.

13. Belhassen B, Glick A, Viskin S. Excellent long-term reproducibility of the electrophysiologic efficacy of quinidine in patients with idiopathic ventricular fibrillation and Brugada syndrome. Pacing Clin Electrophysiol. 2009;32:294-301.

14. Haissaguerre M, Shoda M, Jais P, et al. Mapping and ablation of idiopathic ventricular fibrillation. Circulation. 2002;106:962-7. 